malignancies (OR 1.38, 95\% CI 1.12 to 1.69). Risk of colorectal cancer was significantly decreased (OR $0.76,95 \%$ CI 0.63 to 0.92 ) for COX2 inhibitor usage of more than a year. There were no other significant associations.

Conclusion In this large population-based case-control study, prolonged use of COX2 inhibitors was associated with increased risk of breast and haematological cancers and decreased risk of colorectal cancer. These findings need to be confirmed using other data sources.

\section{4-4.5 ASSOCIATIONS OF ANGIOTENSIN-II RECEPTOR BLOCKERS AND ACE INHIBITORS WITH ALZHEIMER'S DISEASE: A NESTED CASE-CONTROL STUDY WITHIN THE UK GENERAL PRACTICE RESEARCH DATABASE}

doi:10.1136/jech.2011.142976b.28

${ }^{1} \mathrm{~N}$ Davies, * 1,2P Kehoe, ${ }^{1} Y$ Ben-Shlomo, ${ }^{1} \mathrm{R}$ Martin. ${ }^{1}$ University of Bristol, Bristol, UK; ${ }^{2}$ Frenchay Hospital, Bristol, UK

Objectives To investigate whether angiotensin II receptor blockers (ARBs) and ACE inhibitors (ACE-Is) are more strongly associated with Alzheimer's disease (AD) than other anti-hypertensive drugs. Methods Nested case-control analysis within the UK general practice research database ( $\mathrm{n} \approx 10$ million), with prospectively recorded anti-hypertensive prescribing data. Cases aged $\geq 60$ years and diagnosed between 1997 and 2008 (5797 with $\mathrm{AD}, 2186$ with vascular dementia, 1214 with unspecified / other dementia) were matched to up to four controls by age, general practice and gender. We computed $\mathrm{ORs}$ and dose response effects for $\mathrm{AD}$, vascular and unspecified / other dementia, comparing those prescribed ARBs or ACE-Is for at least 6 months with patients prescribed other anti-hypertensives. We controlled for matching factors, co-morbidities, smoking status, an area measure of socioeconomic status, consultation rate and blood pressure and accounted for reverse causality by introducing time-lags of up to 8 years prior to diagnosis / index date.

Results Patients diagnosed with $\mathrm{AD}$, vascular and unspecified / other dementia had fewer prescriptions for ARBs and ACE-Is. Inverse associations with $\mathrm{AD}$ were strongest for $\mathrm{ARBs}$ (OR 0.47, 95\% CI 0.37 to 0.58 ) compared with ACE-Is (OR 0.76, 95\% CI 0.69 to 0.84 ) ( $p$ difference $<0.001$ ). Associations of $A R B s$ with $A D$ were stronger than for vascular dementia ( $p$ difference=0.01) and unspecified / other dementia ( $p$ difference $=0.23$ ). There were inverse dose-response relationships between $\mathrm{ARBs}$ and ACE-Is with $\mathrm{AD}$ (both $p$ trend $<0.01$ ). The inverse association of ACE-Is with $A D$ diminished when using longer time lags but the $A R B-A D$ association persisted.

Conclusions Patients with $\mathrm{AD}$ were around half as likely to be prescribed ARBs. Further randomised controlled trial evidence is required to rigorously test these findings.

\section{4-4.6 ROLE OF MEDICAL FACTORS IN THE AETIOLOGY OF UPPER AERODIGESTIVE TRACT CANCERS IN EUROPE: THE ARCAGE STUDY}

doi:10.1136/jech.2011.142976b.29

\begin{abstract}
${ }^{1} \mathrm{~T}$ Macfarlane, ${ }^{*} \mathrm{G} \mathrm{J}$ Macfarlane, ${ }^{2} \mathrm{M}$ Marron, ${ }^{3} \mathrm{P}$ Brennan, ${ }^{3} \mathrm{ARCAGE}$ Collaboration. ${ }^{1}$ University of Aberdeen, Aberdeen, UK; ${ }^{2}$ University Medical Center of the Johannes Gutenberg University, Mainz, Germany; ${ }^{3}$ International Agency for Research on Cancer, Lyon, France
\end{abstract}

Background Cancer of the upper aerodigestive tract (UADT) (oral cavity, pharynx, larynx and oesophagus) is, globally, the fourth most common cancer and cause of cancer mortality. In addition to established risk factors such as tobacco and alcohol consumption, other risk factors were suggested, including human papillomavirus infection.
Objective To investigate the role of medical history (skin warts / verrucae; Candida albicans / thrush; herpetic lesions / cold sores; heartburn; regurgitation) and medication (for heartburn; for regurgitation; aspirin) use in UADT cancer risk.

Methods A case-control study conducted in 10 European countries. Results There were 1779 cases of UADT cancer (all SCC) and 1993 controls. Having had warts / verrucae and history of Candida / thrush infection protective for UADT cancers (OR $0.80,95 \% \mathrm{CI}$ (0.68 to 0.94 ) and 0.73 (0.60 to 0.89 ), respectively) but there was no association with herpetic lesions.

Neither symptoms of gastro-oesophageal reflux (heartburn or regurgitation) nor medication for associated symptoms were associated with risk of UADT cancer. When considered by sub-site, regurgitation was associated with a non-significant increased risk for cancer of the oesophagus (1.47; 0.98 to 2.21 ).

Regular aspirin use (at least once a week for a year) was not associated with risk of UADT cancer. When considered by sub-site, it had protective effect for cancer of oesophagus $(0.51 ; 0.28$ to 0.96$)$ and non-significant protective effect for cancers of hypopharynx (0.53; 0.28 to 1.02$)$ and larynx (0.74; 0.54 to 1.01$)$.

Conclusion There is conflicting evidence regarding association between medical history and medication use and UADT cancer risk.

\subsection{PREVENTING CHRONIC DISEASE LOCALLY AND GLOBALLY: DELIVERY OF PREVENTION INTERVENTION VIA THE SUPERCOURSE}

\section{Chair: Prof. Ronald LaPorte, USA \\ 04-5.1 TelePreVentive Medicine AS THE FUTURE OF EPIDEMIOLOGY AND DISEASE PREVENTION}

doi:10.1136/Jech.2011.142976b.30

R LaPorte.* University of Pittsburgh, Pittsburgh, Pennsylvania, USA

The problem of chronic disease around the world can be improved only through the means of prevention. Effective prevention of chronic disease cannot be achieved without what we call "Telepreventive Medicine." The fundamental underlying concept is that information about disease prevention should be distributed using inexpensive Internet pathways. The birth of the discipline started a few years ago and has been recognised in the The British Medical Journal article. In the global public health arena, the concept of telepreventive medicine is an integral part of the Global Health Network Supercourse project, a global online library of nearly 5000 lectures and a network of 48000 individuals in 174 countries. Telepreventive medicine has a great potential to improve global health, in both communicable and non-communicable diseases. For example, utilising the power of the Supercourse network and telepreventive medicine, Supercourse team was able to distribute information about H1N1 infection even before it made it to news media. We must differentiate telepreventive medicine from telemedicine. Telemedicine is designed to "cure" and it is expensive (like "telesurgery", it is unlikely that telemedicine can have any effect on global health as it does not reach too many people. In contrast with telepreventive medicine we can reach millions with the prevention message. Through the Supercourse, telepreventive medicine epidemiology and new mobile global health approaches, information can be shared more rapidly with all students through their instructors.

Visit the Telepreventive Medicine Supercourse lecture at http:// www.pitt.edu/ super1/lecture/lec10431/index.htm. 